\title{
Comparative Evaluation of varying Photo-polymerization Time on Shear Bond Strength and Microleakage of Four Orthodontic Adhesives: An in vitro Study
}

\author{
${ }^{1}$ Prerana N Zanke, ${ }^{2}$ Vivek Patni, ${ }^{3}$ Marshal Maskarenj
}

\begin{abstract}
Introduction: High early bond strength, extended working time for optimal bracket placement, and easy cleanup of excess adhesive are few advantages of visible light-cure orthodontic adhesives. However, the shorter duration of polymerization may lead to polymer shrinkage and eventually microleakage.

Objective: To observe and evaluate shear bond strength (SBS) and microleakage of orthodontic brackets bonded to enamel with four commercially available orthodontic adhesives, cured only from incisal direction at various polymerization times.

Materials and methods: A total of 160 bovine incisors were randomly assigned to four groups according to adhesive used. Group I: bonded with Transbond XT (3M Unitek ${ }^{\mathrm{TM}}$, USA); group II: Enlight (Ormco, USA); group III: Light Bond (Reliance Ortho, USA); and group IV: Discover (Prime Dental, USA). Each group was further divided into four subgroups of $A, B, C$, and $\mathrm{D}$ according to the polymerization duration of $5,10,15$, and 20 seconds respectively. The bonded teeth were immersed in dye solution. Brackets were then subjected to SBS test on a Universal testing machine. Surface microleakage was observed with the help of optical stereomicroscope.
\end{abstract}

Results: The SBS was obtained within the range of clinically accepted values, with curing time for Enlight at 5 seconds, Transbond XT and Discover at 15 seconds, and Light Bond at 20 seconds. However, Enlight was demonstrated to provide optimum SBS at least curing time: Minimum duration of 5 seconds achieved adequate SBS $\sim 11 \mathrm{MPa}$ SBS for each of Transbond XT and Enlight. The surface microleakage observed is statistically insignificant among the groups.

Conclusion: From a clinical perspective, a composite resin that needs minimum curing time without compromising on the bond strength is most advantageous. The results for SBS tests showed a better performance for Enlight as compared with the other adhesives: it reached its bond strength of optimum value at curing time of 5 seconds.

Keywords: Microleakage, Orthodontic adhesives, Shear bond strength.

\footnotetext{
${ }^{1}$ Postgraduate Student, ${ }^{2}$ Professor, ${ }^{3}$ Research Scholar

1,2Department of Orthodontics and Dentofacial Orthopedics Mahatma Gandhi Mission's Dental College and Hospital, Navi Mumbai, Maharashtra, India

${ }^{3}$ Department of Energy Science and Engineering, Indian Institute of Technology Bombay, Mumbai, Maharashtra, India

Corresponding Author: Prerana N Zanke, Postgraduate Student Department of Orthodontics and Dentofacial Orthopedics Mahatma Gandhi Mission's Dental College and Hospital, Navi Mumbai, Maharashtra, India, Phone: +919867903356, e-mail: dr.preranaz@gmail.com
}

How to cite this article: Zanke PN, Patni V, Maskarenj M. Comparative Evaluation of varying Photo-polymerization Time on Shear Bond Strength and Microleakage of Four Orthodontic Adhesives: An in vitro Study. J Contemp Dent 2017;7(3):156-160.

Source of support: Nil

Conflict of interest: None

\section{INTRODUCTION}

The development of bisphenol A-glycidyl methacrylate resins revolutionized adhesive dentistry, and these materials are now an integral part of many forms of treatment. High early bond strength, ${ }^{1}$ extended working time for optimal bracket placement, and easier cleanup of excess adhesive are the advantages of visible light cure orthodontic adhesives. Light-emitting diode (LED) curing lights can cure the composite bonding resins in shorter time owing to higher intensity of the curing light. ${ }^{2}$ Studies have shown that 3 to 5 seconds of light curing for adhesives beneath the brackets is adequate. ${ }^{3}$ From a clinician's point of view, the composite bonding resins that need minimum curing time without compromising on the bond strength and depth of curing are most advantageous. Shorter curing times are often associated with polymerization shrinkage leading to microleakage. ${ }^{4}$ Thus, the purpose of this study is to evaluate and compare variation in polymerization time on the SBS of four orthodontic adhesives and study of microleakage.

\section{RATIONALE}

While contemporary orthodontic bonding materials have the required SBS to merit effective use in clinical conditions, the method and duration of effecting the photopolymerization, as suggested by individual manufacturers, tend to differ a lot.

Duration of cure and recommended surface or surfaces to effect the cure are all a matter of contention. Maintaining the bracket at the desired location on the tooth for the minimum time without compromising on the availability of the necessary SBS to merit effective clinical use is the demand of the occasion. Incisal or occlusal surface of every orthodontic attachment is the most consistent area of effective exposure to photopolymerization notwithstanding the rotations or crowding 
exhibited in most malocclusions. The present study aims to assess four commercial adhesives used for bonding orthodontic attachments over varying curing times to arrive at optimizing the variables studied to act as a guide for the clinician.

Arguably, one of the other disadvantages of using visible light-cured adhesives in orthodontic bonding is the shrinkage during polymerization, which may lead to marginal gaps and eventually marginal leakage at tooth-adhesive interface. This study aims to evaluate and compare the effect of varying photopolymerization time on SBS and microleakage of four orthodontic adhesives.

\section{MATERIALS AND METHODS}

- Four commercially available orthodontic adhesives:

- Enlight (Ormco, USA)

- Light Bond (Reliance Ortho, USA)

- Transbond XT (3M Unitek $\left.{ }^{\mathrm{TM}}, \mathrm{USA}\right)$

- Discover (Prime Dental, USA)

- Cold cure acrylic resin, Acralyn "R" (Asian Acrylates, Mumbai, India)

- Caries free and unfractured bovine incisors collected over a period of 48 hours

- Metal brackets (3M Unitek, Monrovia California, USA) for maxillary central incisors with slot size $0.022^{\prime \prime} \times$ 0.028 "; McLaughin, Bennet, Trevisi (MBT) prescription

- $37 \%$ phosphoric acid gel: PROetch etchant (SS White, USA)

- Disinfectant: $0.1 \%$ thymol solution

- Custom-made methylene blue dye $(0.5 \%$ solution in deionized water $\mathrm{v} / \mathrm{v}$ )

The bovine mandibular incisors ${ }^{5,6}$ were procured from a government-recognized abattoir (Deonar, Mumbai) and gently scrubbed under constant flow of saline solution to detach debris and adhering periodontal ligament tissues. An ultrasonic scaler was used to remove the hard deposits from the tooth surface, before storing in plastic bottle containing $0.1 \%$ thymol solution (SD Finechem Ltd, Mumbai, India) at 0 to $14^{\circ} \mathrm{C}$ to prevent bacterial growth. Teeth samples were washed with distilled water to eliminate traces of thymol, prior to the experimentation; and horizontal notches for retention purpose were made on the root surface using a bur. The entire root of each tooth was embedded in a block made of cold cure acrylic resin extended until the cementoenamel junction. As such, the entire tooth crown portion was left exposed. The acrylic block dimensions were $2.5 \mathrm{~cm}(\mathrm{~L}) \times 1.5 \mathrm{~cm}(\mathrm{~B})$ $\times 4.5 \mathrm{~cm}(\mathrm{H})$ as demonstrated in Figure 1 .

The specimens were then randomly divided into four main groups based on the adhesive used for bracket bonding: Transbond XT (3M Unitek ${ }^{\mathrm{TM}}$, USA), Enlight (Ormco, USA), Discover (Prime Dental, USA), and Light Bond (Reliance Ortho, USA) and further divided into four subgroups according to the polymerization time of 5, 10, 15 , and 20 seconds. Each of these 16 subgroups, thus, had a sample size of 10 acrylic embedded teeth.

Exposed crowns were polished with a bristle brush and a fluoride-free slurry of pumice and water, and the accurate position for bracket placement marked with a Boone's Bracket positioning gauge $(\sim 5 \mathrm{~mm}$ from the incisal edge) postrinsing and drying of the samples. Samples were then etched with $37 \%$ phosphoric acid gel (PROetch, SS White) and again rinsed-dried until a frosty white appearance was appreciated on the surface. Samples were then coated with primer and thinned with a jet of air from an air water syringe. The brackets were loaded with one of the four types of adhesives and placed to fully contact the tooth, depending on the group allocated to the certain sample. Excess flash was removed from the sample-bracket interface.
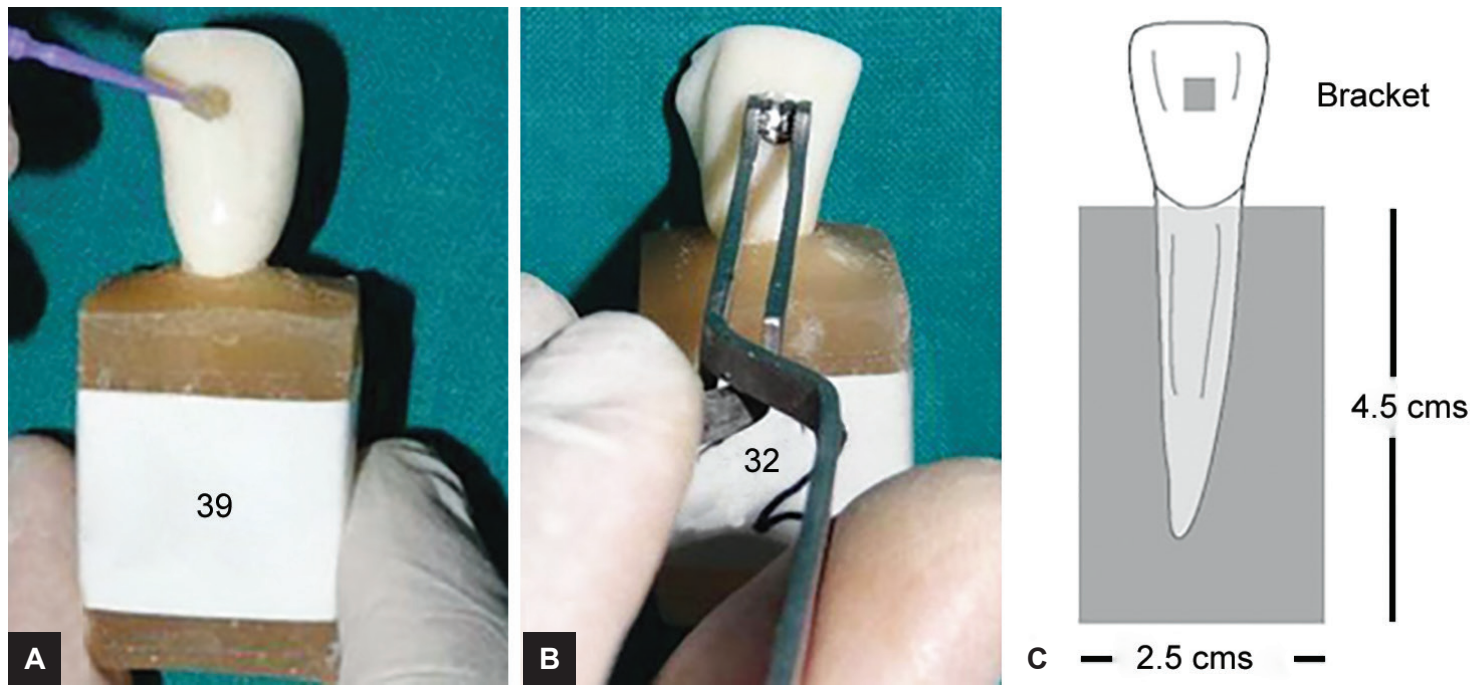

Figs $1 \mathrm{~A}$ to $\mathrm{C}$ : Acrylic-embedded tooth sample: (A) Etching; and (B) bracket placement; and (C) schematic 

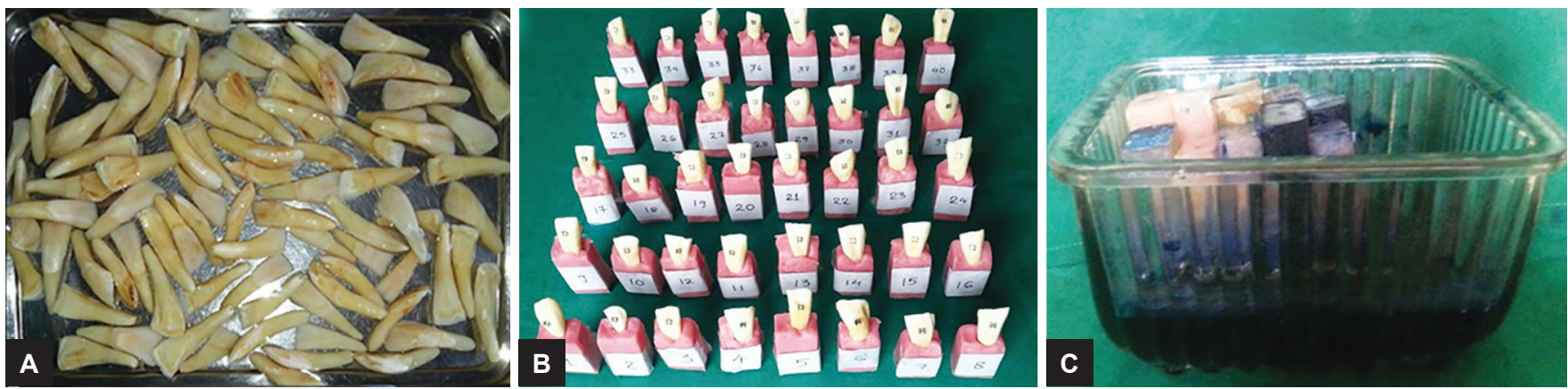

Figs 2A to C: Bovine teeth samples: (A) Crude; (B) placed in acrylic blocks; and (C) placed in dye

Photopolymerization was carried out for 5, 10, 15, or 20 seconds for each of the adhesives respectively, from the incisal surface based on the subgroup allocated for the sample. The specimens (Fig. 2) were then placed in a container of custom-made solution of methylene blue dye and deionized water for 6 days.?

After staining, the brackets were subjected to the testing of SBS with a Universal Testing Machine (UTM), Instron. The specimens were oriented as such the bracketbase was parallel to the blade delivering the debonding force. Debonding force was applied for effecting shearing of the bracket base in an incisogingival direction at a cross-head speed of $1 \mathrm{~mm} / \mathrm{min}$ as demonstrated in Figure 3 . The results were recorded in $\mathrm{kgF}$ and were later converted to MPa.

Debonded specimens were then randomly examined by measuring deepest dye penetration on the tooth surface perpendicular to the bracket margin and rounding the nearest $0.2 \mathrm{~mm}$. Observations were made at the tooth surface with $50 \times$ magnification with a grid with an optical stereomicroscope to observe the microleakage at the sample.

\section{Evaluation Parameters}

The SBS was measured in $\mathrm{kgF}$ and converted into MPa for each of the 160 samples and the data were statistically analyzed with an unpaired t-test.

The microleakage was observed by penetration of the dye on the tooth surface with the help of grid of squares of dimension $1,000 \mu \mathrm{m}$. Each square was allotted a score of 1 from each of incisal, gingival, mesial, and distal surfaces.

\section{RESULTS}

The mean SBS for various adhesives for various curing times are tabulated and presented in Table 1 along with

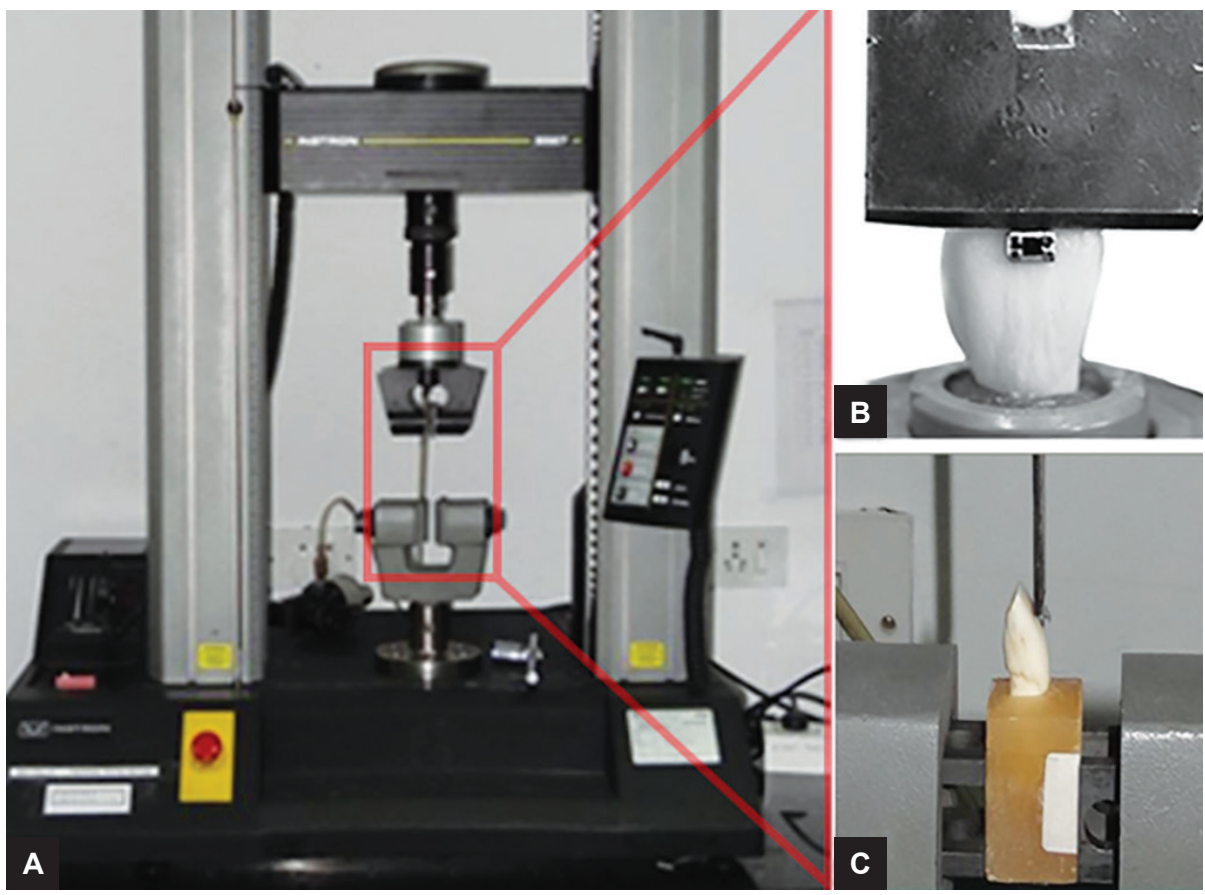

Figs 3A to C: Debonding forces applied on the tooth-bracket interface using universal testing machine (UTM): (A) UTM with fixed attachment for acrylic block placement and movable blade; (B) frontal view of blade at the edge of bracket base; and (C) side view 
Comparative Evaluation of varying Photo-polymerization Time

Table 1: Mean SBS and standard deviation for various adhesives for various curing times

\begin{tabular}{|c|c|c|c|c|c|c|c|c|}
\hline \multirow[b]{2}{*}{$\begin{array}{l}\text { Curing time } \\
\text { Adhesive }\end{array}$} & \multicolumn{2}{|c|}{$5 \mathrm{sec}$} & \multicolumn{2}{|c|}{$10 \mathrm{sec}$} & \multicolumn{2}{|c|}{$15 \mathrm{sec}$} & \multicolumn{2}{|c|}{$20 \mathrm{sec}$} \\
\hline & Mean (MPa) & $\begin{array}{l}\text { Standard } \\
\text { deviation }\end{array}$ & Mean (MPa) & $\begin{array}{l}\text { Standard } \\
\text { deviation }\end{array}$ & Mean (MPa) & $\begin{array}{l}\text { Standard } \\
\text { deviation }\end{array}$ & Mean (MPa) & $\begin{array}{l}\text { Standara } \\
\text { deviation }\end{array}$ \\
\hline Transbond XT & 6.49 & 1.976 & 6.80 & 3.615 & 11.25 & 4.751 & 13.70 & 4.615 \\
\hline Enlight & 10.87 & 4.196 & 11.16 & 2.730 & 15.40 & 2.054 & 22.86 & 5.333 \\
\hline Discover & 4.68 & 1.570 & 7.66 & 4.023 & 7.91 & 1.919 & 10.64 & 11.893 \\
\hline Light Bond & 3.39 & 1.062 & 5.49 & 1.061 & 8.14 & 3.479 & 11.27 & 3.055 \\
\hline
\end{tabular}

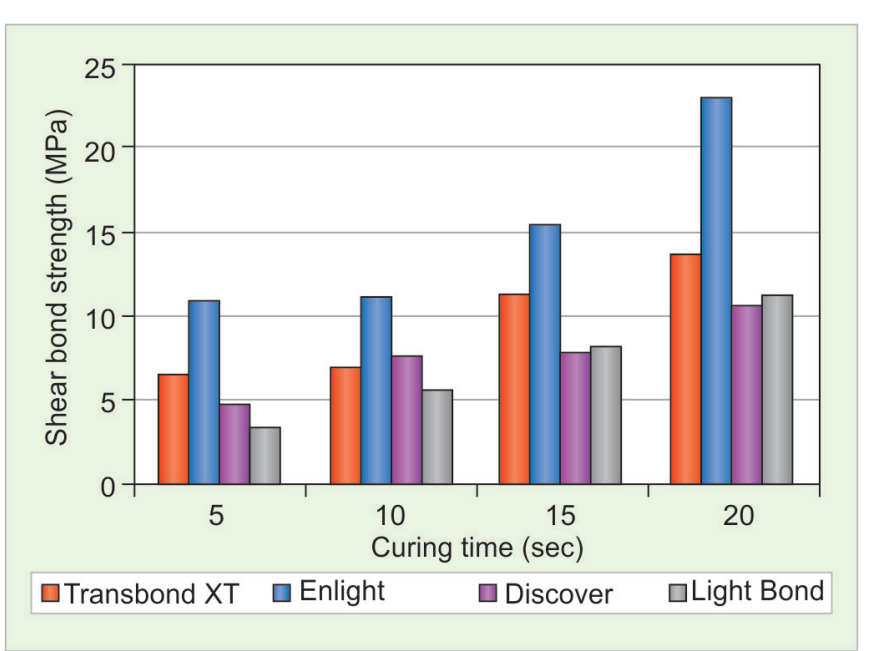

Graph 1: Mean SBS for various adhesives at various curing times standard deviation (SD) in values through the sample size of 10 per subgroup.

Enlight (Ormco, USA) demonstrated to provide optimum SBS of $10.87 \mathrm{MPa}$ at a minimum curing time of 5 seconds. However, at low curing time, the high SD showed a lesser consistency between the samples. Low SD between sample readings showed a consistent performance at low curing time for almost all other adhesives. At a higher curing time, however, Enlight (Ormco, USA) showed a better performance as compared with Discover (Prime Dental, USA) with a huge inconsistency between samples at 20 seconds. Graph 1 shows a graphical representation of the data presented in Table 1.

Two-factor analysis of variance (ANOVA) was performed on the data to calculate the effect of adhesives and curing time on the SBS, individually and in tandem.
Table 2 shows the tabulated value for the two-factor ANOVA.

At a confidence interval (CI) of $95 \%$, the adhesive brand showed a very low p-value, and as such the null hypothesis had to be discarded: The brand of adhesive used had a significant impact on the SBS in these experimental data. Similarly, the curing time showed a very low $p$-value signifying the impact of curing time on the resulting SBS. However, the $p$-value for interaction between the two factors had a p-value of $>0.05$ demonstrating that the two factors simultaneously do not play a significant role at a $\mathrm{CI}$ of $95 \%$.

Acceptable SBS was obtained within the range of each of manufacturer's recommendation, i.e., Enlight for 5 seconds, Transbond XT and Discover for 15 seconds, and Light Bond for 20 seconds.

\section{CONCLUSION}

Selection of orthodontic adhesive system may play an important role in achieving adequate SBS with minimum curing time. The most advantageous composite bonding resins for clinical purposes are those that need minimum curing time without compromising on bond strength, depth of curing, and microleakage. Enlight (Ormco) was demonstrated to provide optimum SBS at low curing time: Minimum duration of 5 seconds achieved adequate SBS 11 MPa.

Regardless of the promising results presented in this study, care should be taken in the interpretation of the results, as well as applying into clinical situations as in vitro bond strengths are usually higher than those obtained in vivo.

Table 2: ANOVA: two factor with replication at $95 \% \mathrm{Cl}$

\begin{tabular}{lllllll}
\hline Source of variation & $S S$ & $d f$ & $M S$ & $f$-value & $p$-value & $F$ crit \\
\hline Adhesive brand (a) & 1584.203 & 3 & 528.0675 & 27.60854 & $3.65 \mathrm{E}-14$ & 2.667443 \\
Curing time (b) & 1595.376 & 3 & 531.792 & 27.80327 & $3.04 \mathrm{E}-14$ & 2.667443 \\
Interaction of (a) and (b) & 238.4072 & 9 & 26.48969 & 1.38494 & 0.200062 & 1.94545 \\
Within & 2754.282 & 144 & 19.12696 & & & \\
\hline
\end{tabular}

The effect is not significant if $p$-value is $>0.05$ at $95 \% \mathrm{Cl}$ 


\section{REFERENCES}

1. Eliades T, Viazis AD, Eliades G. Bonding of ceramic brackets to enamel: morphologic and structural considerations. Am J Orthod Dentofacial Orthop 1991 Apr;99(4): 369-375

2. Swanson T, Dunn WJ, Childers DE, Taloumis LJ. Shear bond strength of orthodontic brackets bonded with light-emitting diode curing units at various polymerization times. Am J Orthod Dentofacial Orthop 2004 Mar;125(3):337-341.

3. Oesterie LJ, Newman SM, Shellhart WC. Rapid curing of bonding composite with a xenon plasma arc light. Am J Orthod Dentofacial Orthop 2001 Jun;119(6):610-616.
4. James JW, Miller BH, English JD, Tadlock LP, Buschang PH. Effects of high-speed curing devices on shear bond strength and microleakage of orthodontic brackets. Am J Orthod Dentofacial Orthop 2003 May;123(5):555-561.

5. Oesterle LJ, Shellhart WC, Belanger GK. The use of bovine enamel in bonding studies. Am J Orthod Dentofacial Orthop 1998 Nov;114(5):514-523.

6. Nakamichi I, Iwaku M, Fusayama T. Bovine teeth as possible substitutes in the adhesion test. J Dent Res 1983 Oct;62(10): 1076-1081.

7. Ulker M, Uysal T, Romoglu SI, Ertas H. Microleakage under orthodontic bracket using high intensity curing lights. Angle Orthod 2009 Jan;79(1):144-149. 